\title{
Right for the Right Reasons: Training Differentiable Models by Constraining their Explanations
}

\author{
Andrew Slavin Ross, Michael C. Hughes, and Finale Doshi-Velez \\ Paulson School of Engineering and Applied Sciences, Harvard University, Cambridge, MA 02138, USA \\ andrew_ross@g.harvard.edu,mchughes@seas.harvard.edu, finale@seas.harvard.edu
}

\begin{abstract}
Neural networks are among the most accurate supervised learning methods in use today. However, their opacity makes them difficult to trust in critical applications, especially if conditions in training may differ from those in test. Recent work on explanations for black-box models has produced tools (e.g. LIME) to show the implicit rules behind predictions. These tools can help us identify when models are right for the wrong reasons. However, these methods do not scale to explaining entire datasets and cannot correct the problems they reveal. We introduce a method for efficiently explaining and regularizing differentiable models by examining and selectively penalizing their input gradients. We apply these penalties both based on expert annotation and in an unsupervised fashion that produces multiple classifiers with qualitatively different decision boundaries. On multiple datasets, we show our approach generates faithful explanations and models that generalize much better when conditions differ between training and test.
\end{abstract}

\section{Introduction}

High-dimensional real-world datasets are often full of ambiguities. When we train classifiers on such data, it is frequently possible to achieve high accuracy using classifiers with qualitatively different decision boundaries. To narrow down our choices and encourage robustness, we usually employ regularization techniques (e.g. encouraging sparsity or small parameter values). We also structure our models to ensure domain-specific invariances (e.g. using convolutional neural nets when we would like the model to be invariant to spatial transformations). However, these solutions do not address situations in which our training dataset contains subtle confounds or differs qualitatively from our test dataset. In these cases, our model may fail to generalize no matter how well it is tuned.

Such generalization gaps are of particular concern for uninterpretable models such as neural networks, especially in sensitive domains. For example, [Caruana et al., 2015] describe a model intended to prioritize care for patients with pneumonia. The model was trained to predict hospital readmission risk using a dataset containing attributes of patients hospitalized at least once for pneumonia. Counterintuitively, the model learned that the presence of asthma was a negative predictor of readmission, when in reality pneumonia patients with asthma are at a greater medical risk. This model would have presented a grave safety risk if used in production. This problem occurred because the outcomes in the dataset reflected not just the severity of patients' diseases but the quality of care they initially received, which was higher for patients with asthma.

This case and others like it have motivated recent work in interpretable machine learning, where algorithms provide explanations for domain experts to inspect for correctness before trusting model predictions. However, there has been limited work in optimizing models to find not just the right prediction but also the right explanation. Toward this end, this work makes the following contributions:

- We confirm empirically on several datasets that input gradient explanations match state of the art sample-based explanations (e.g. LIME [Ribeiro, 2016]).

- Given annotations about incorrect explanations for particular inputs, we efficiently optimize the classifier to learn alternate explanations (to be right for better reasons).

- When annotations are not available, we sequentially discover classifiers with similar accuracies but qualitatively different decision boundaries for domain experts to inspect for validity.

\subsection{Related Work}

We first define several important terms in interpretable machine learning. All classifiers have implicit decision rules for converting an input into a decision, though these rules may be opaque. A model is interpretable if it provides explanations for its predictions in a form humans can understand; an explanation provides reliable information about the model's implicit decision rules for a given prediction. In contrast, we say a machine learning model is accurate if most of its predictions are correct, but only right for the right reasons if the implicit rules it has learned generalize well and conform to domain experts' knowledge about the problem.

Explanations can take many forms [Keil, 2006] and evaluating the quality of explanations or the interpretability of a 
model is difficult [Lipton, 2016; Doshi-Velez and Kim, 2017]. However, within the machine learning community recently there has been convergence [Lundberg and Lee, 2016] around local counterfactual explanations, where we show how perturbing an input $x$ in various ways will affect the model's prediction $\hat{y}$. This approach to explanations can be domain- and model-specific (e.g. "annotator rationales" used to explain text classifications in [Li et al., 2016; Lei et al., 2016; Zhang et al., 2016]). Alternatively, explanations can be model-agnostic and relatively domain-general, as exemplified by LIME (Local Interpretable Model-agnostic Explanations, [Ribeiro et al., 2016; Singh et al., 2016]) which trains and presents local sparse models of how predictions change when inputs are perturbed.

The per-example perturbing and fitting process used in models such as LIME can be computationally prohibitive, especially if we seek to explain an entire dataset during each training iteration. If the underlying model is differentiable, one alternative is to use input gradients as local explanations ([Baehrens et al., 2010] provides a particularly good introduction; see also [Selvaraju et al., 2016; Simonyan et al., 2013; Li et al., 2015; Hechtlinger, 2016]). The idea is simple: the gradients of the model's output probabilities with respect to its inputs literally describe the model's decision boundary (see Figure 1). They are similar in spirit to the local linear explanations of LIME but much faster to compute.

Input gradient explanations are not perfect for all usecases-for points far from the decision boundary, they can be uniformatively small and do not always capture the idea of salience (see discussion and alternatives proposed in [Shrikumar et al., 2016; Bach et al., 2015; Montavon et al., 2017; Sundararajan et al., 2016; Shrikumar et al., 2017; Fong and Vedaldi, 2017]). However, they are exactly what is required for constraining the decision boundary. In the past, [Drucker and Le Cun, 1992] showed that applying penalties to input gradient magnitudes can improve generalization; to our knowledge, our application of input gradients to constrain explanations and find alternate explanations is novel.

More broadly, none of the works above on interpretable machine learning attempt to optimize explanations for correctness. For SVMs and specific text classification architectures, there

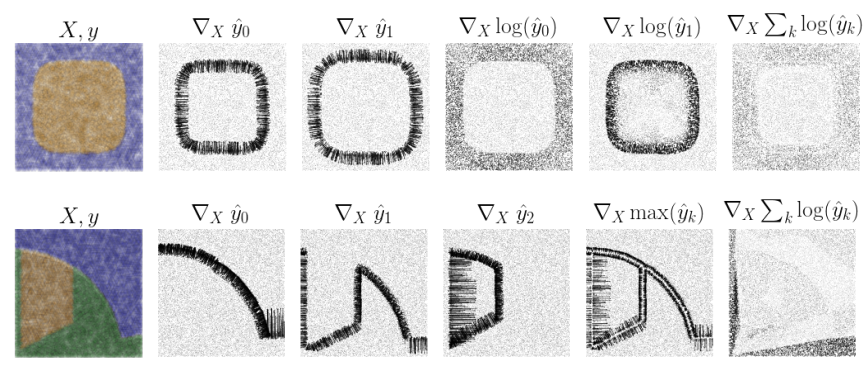

Figure 1: Input gradients lie normal to the model's decision boundary. Examples above are for simple, 2D, two- and three-class datasets, with input gradients taken with respect to a two hidden layer multilayer perceptron with ReLU activations. Probability input gradients are sharpest near decision boundaries, while log probabilities input gradients are more consistent within decision regions. The sum of log probability gradients contains information about the full model. exists work on incorporating human input into decision boundaries in the form of annotator rationales [Zaidan et al., 2007; Donahue and Grauman, 2011; Zhang et al., 2016]. Unlike our approach, these works are either tailored to specific domains or do not fully close the loop between generating explanations and constraining them.

\subsection{Background: Input Gradient Explanations}

Consider a differentiable model $f$ parametrized by $\theta$ with inputs $X \in \mathbb{R}^{N \times D}$ and probability vector outputs $f(X \mid \theta)=$ $\hat{y} \in \mathbb{R}^{N \times K}$ corresponding to one-hot labels $y \in \mathbb{R}^{N \times K}$. Its input gradient is given by $f_{X}\left(X_{n} \mid \theta\right)$ or $\nabla_{X} \hat{y}_{n}$, which is a vector normal to the model's decision boundary at $X_{n}$ and thus serves as a first-order description of the model's behavior near $X_{n}$. The gradient has the same shape as each vector $X_{n}$; largemagnitude values of the input gradient indicate elements of $X_{n}$ that would affect $\hat{y}$ if changed. We can visualize explanations by highlighting portions of $X_{n}$ in locations with high input gradient magnitudes.

\section{Our Approach}

We wish to develop a method to train models that are right for the right reasons. If explanations faithfully describe a model's underlying behavior, then constraining its explanations to match domain knowledge should cause its underlying behavior to more closely match that knowledge too. We first describe how input gradient-based explanations lend themselves to efficient optimization for correct explanations in the presence of domain knowledge, and then describe how they can be used to efficiently search for qualitatively different decision boundaries when such knowledge is not available.

\subsection{Loss Functions that Constrain Explanations}

When constraining input gradient explanations, there are two basic options: we can either constrain them to be large in relevant areas or small in irrelevant areas. However, because input gradients for relevant inputs in many models should be small far from the decision boundary, and because we do not know in advance how large they should be, we opt to shrink irrelevant gradients instead.

Formally, we define an annotation matrix $A \in\{0,1\}^{N \times D}$, which are binary masks indicating whether dimension $d$ should be irrelevant for predicting observation $n$. We would like $\nabla_{X} \hat{y}$ to be near 0 at these locations. To that end, we optimize a loss function $L(\theta, X, y, A)$ of the form

$$
\begin{aligned}
& L(\theta, X, y, A)=\underbrace{\sum_{n=1}^{N} \sum_{k=1}^{K}-y_{n k} \log \left(\hat{y}_{n k}\right)}_{\text {Right answers }} \\
& +\underbrace{\lambda_{1} \sum_{n=1}^{N} \sum_{d=1}^{D}\left(A_{n d} \frac{\partial}{\partial x_{n d}} \sum_{k=1}^{K} \log \left(\hat{y}_{n k}\right)\right)^{2}}_{\text {Right reasons }}+\underbrace{\lambda_{2} \sum_{i} \theta_{i}^{2}}_{\text {Regular }},
\end{aligned}
$$

which contains familiar cross entropy and $\theta$ regularization terms along with a new regularization term that discourages the input gradient from being large in regions marked by $A$. 
This term has a regularization parameter $\lambda_{1}$ which should be set such that the "right answers" and "right reasons" terms have similar orders of magnitude; see Appendix A for more details. Note that this loss penalizes the gradient of the $\log$ probability, which performed best in practice, though in many visualizations we show $f_{X}$, which is the gradient of the predicted probability itself. Summing across classes led to slightly more stable results than using the predicted class log probability $\max \log \left(\hat{y}_{k}\right)$, perhaps due to discontinuities near the decision boundary (though both methods were comparable). We did not explore regularizing input gradients of specific class probabilities, though this would be a natural extension.

Because this loss function is differentiable with respect to $\theta$, we can easily optimize it with gradient-based optimization methods. We do not need annotations (nonzero $A_{n}$ ) for every input in $X$, and in the case $A=0^{N \times D}$, the explanation term has no effect on the loss. At the other extreme, when $A$ is a matrix of all $1 \mathrm{~s}$, it encourages the model to have small gradients with respect to its inputs; this can improve generalization on its own [Drucker and Le Cun, 1992]. Between those extremes, it biases our model against particular implicit rules.

This penalization approach enjoys several desirable properties. Alternatives that specify a single $A_{d}$ for all examples presuppose a coherent notion of global feature importance, but when decision boundaries are nonlinear many features are only relevant in the context of specific examples. Alternatives that simulate perturbations to entries known to be irrelevant (or to determine relevance as in [Ribeiro et al., 2016]) require defining domain-specific perturbation logic; our approach does not. Alternatives that apply hard constraints or completely remove elements identified by $A_{n d}$ miss the fact that the entries in $A$ may be imprecise even if they are human-provided. Thus, we opt to preserve potentially misleading features but softly penalize their use.

\subsection{Find-Another-Explanation: Discovering Many Possible Rules without Annotations}

Although we can obtain the annotations $A$ via experts as in [Zaidan et al., 2007], we may not always have this extra information or know the "right reasons." In these cases, we propose an approach that iteratively adapts $A$ to discover multiple models accurate for qualitatively different reasons; a domain expert could then examine them to determine which is the right for the best reasons. Specifically, we generate a "spectrum" of models with different decision boundaries by iteratively training models, explaining $X$, then training the next model to differ from previous iterations:

$$
\begin{array}{rlrl}
A_{0} & =0, & \theta_{0} & =\underset{\theta}{\arg \min } L\left(\theta, X, y, A_{0}\right), \\
A_{1} & =M_{c}\left[f_{X} \mid \theta_{0}\right], & \theta_{1} & =\underset{\theta}{\arg \min } L\left(\theta, X, y, A_{1}\right), \\
A_{2} & =M_{c}\left[f_{X} \mid \theta_{1}\right] \cup A_{1}, & \theta_{2} & =\underset{\theta}{\arg \min } L\left(\theta, X, y, A_{2}\right), \\
\ldots &
\end{array}
$$

where the function $M_{c}$ returns a binary mask indicating which gradient components have a magnitude ratio (their magnitude divided by the largest component magnitude) of at least $c$ and where we abbreviated the input gradients of the entire training set $X$ at $\theta_{i}$ as $f_{X} \mid \theta_{i}$. In other words, we regularize input gradients where they were largest in magnitude previously. If, after repeated iterations, accuracy decreases or explanations stop changing (or only change after significantly increasing $\lambda_{1}$ ), then we have spanned the space of possible models. All of the resulting models will be accurate, but for different reasons; although we do not know which reasons are best, we can present them to a domain expert for inspection and selection. We can also prioritize labeling or reviewing examples about which the ensemble disagrees. Finally, the size of the ensemble provides a rough measure of dataset redundancy.

\section{Empirical Evaluation}

We demonstrate explanation generation, explanation constraints, and the find-another-explanation method on a toy color dataset and three real-world datasets. In all cases, we used a multilayer perceptron with two hidden layers of size 50 and 30, ReLU nonlinearities with a softmax output, and a $\lambda_{2}=0.0001$ penalty on $\|\theta\|_{2}^{2}$. We trained the network using Adam [Kingma and $\mathrm{Ba}, 2014]$ (with a batch size of 256) and Autograd [Mclaurin et al., 2017]. For most experiments, we used an explanation L2 penalty of $\lambda_{1}=1000$, which gave our "right answers" and "right reasons" loss terms similar magnitudes. More details about cross-validation are included in Appendix A. For the cutoff value $c$ described in Section 2.2 and used for display, we often chose 0.67 , which tended to preserve $2-5 \%$ of gradient components (the average number of qualifying elements tended to fall exponentially with $c$ ). Code for all experiments is available at https://github.com/dtak/rrr.

\subsection{Toy Color Dataset}

We created a toy dataset of $5 \times 5 \times 3$ RGB images with four possible colors. Images fell into two classes with two independent decision rules a model could implicitly learn: whether their four corner pixels were all the same color, and whether their top-middle three pixels were all different colors. Images in class 1 satisfied both conditions and images in class 2 satisfied neither. Because only corner and top-row pixels are relevant, we expect any faithful explanation of an accurate model to highlight them.

In Figure 2, we see both LIME and input gradients identify the same relevant pixels, which suggests that (1) both methods are effective at explaining model predictions, and (2) the model

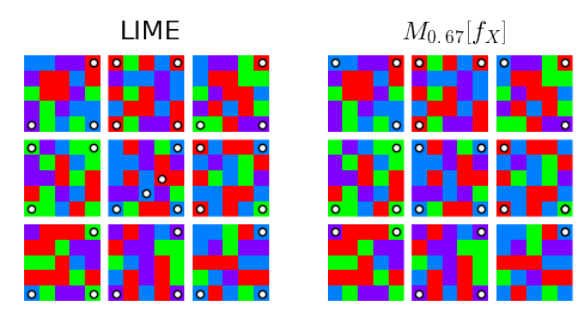

Figure 2: Gradient vs. LIME explanations of nine perceptron predictions on the Toy Color dataset. For gradients, we plot dots above pixels identified by $M_{0.67}\left[f_{X}\right]$ (the top 33\% largest-magnitude input gradients), and for LIME, we select the top 6 features (up to 3 can reside in the same RGB pixel). Both methods suggest that the model learns the corner rule. 

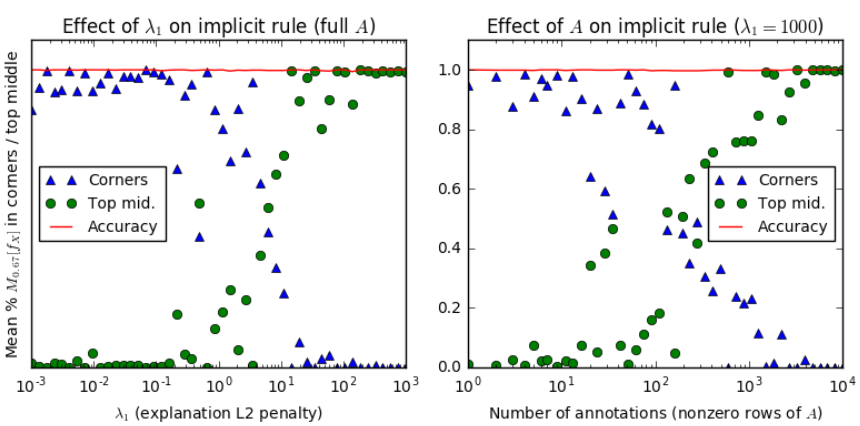

Figure 3: Implicit rule transitions as we increase $\lambda_{1}$ and the number of nonzero rows of $A$. Pairs of points represent the fraction of large-magnitude $(c=0.67)$ gradient components in the corners and top-middle for 1000 test examples, which almost always add to 1 (indicating the model is most sensitive to these elements alone, even during transitions). Note there is a wide regime where the model learns a hybrid of both rules.
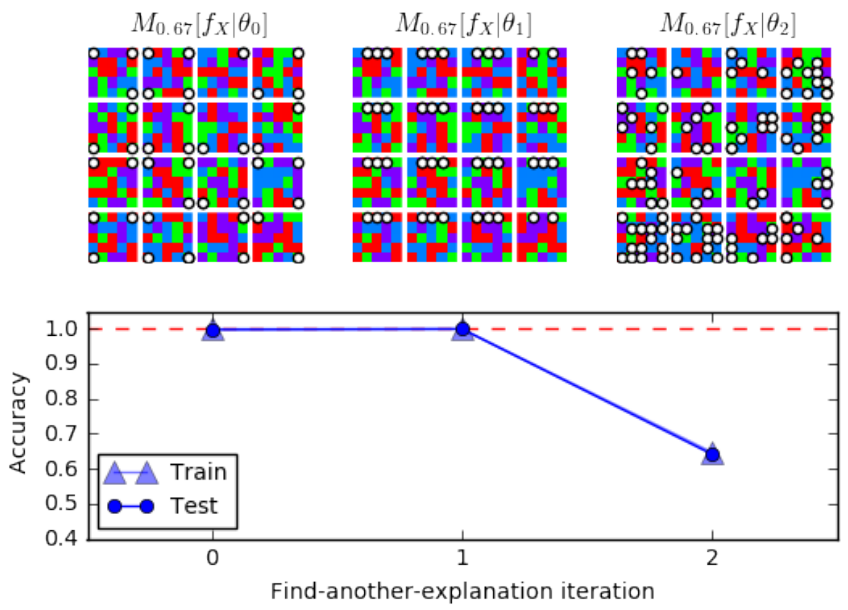

Figure 4: Rule discovery using find-another-explanation method with 0.67 cutoff and $\lambda_{1}=10^{3}$ for $\theta_{1}$ and $\lambda_{1}=10^{6}$ for $\theta_{2}$. Note how the first two iterations produce explanations corresponding to the two rules in the dataset while the third produces very noisy explanations (with low accuracies).

has learned the corner rather than the top-middle rule, which it did consistently across random restarts.

However, if we train our model with a nonzero $A$ (specifically, setting $A_{n d}=1$ for corners $d$ across examples $n$ ), we were able to cause it to use the other rule. Figure 3 shows how the model transitions between rules as we vary $\lambda_{1}$ and the number of examples penalized by $A$. This result demonstrates that the model can be made to learn multiple rules despite only one being commonly reached via standard gradient-based optimization methods. However, it depends on knowing a good setting for $A$, which in this case would still require annotating on the order of $10^{3}$ examples, or $5 \%$ of our dataset (although always including examples with annotations in Adam minibatches let us consistently switch rules with only 50 examples, or $0.2 \%$ of the dataset).

Finally, Figure 4 shows we can use the find-anotherexplanation technique from Sec. 2.2 to discover the other rule without being given $A$. Because only two rules lead to high accuracy on the test set, the model performs no better than random guessing when prevented from using either one (although we have to increase the penalty high enough that this accuracy number may be misleading - the essential point is that after the first iteration, explanations stop changing). Lastly, though not directly relevant to the discussion on interpretability and explanation, we demonstrate the potential of explanations to reduce the amount of data required for training in Appendix B.

\subsection{Real-world Datasets}

To demonstrate real-world, cross-domain applicability, we test our approach on variants of three familiar machine learning text, image, and tabular datasets:

- 20 Newsgroups: As in [Ribeiro et al., 2016], we test input gradients on the alt.atheism vs. soc.religion. christian subset of the 20 Newsgroups dataset [Lichman, 2013]. We used the same twohidden layer network architecture with a TF-IDF vectorizer with 5000 components, which gave us a 94\% accurate model for $A=0$.

- Iris-Cancer: We concatenated all examples in classes 1 and 2 from the Iris dataset with the the first 50 examples from each class in the Breast Cancer Wisconsin dataset [Lichman, 2013] to create a composite dataset $X \in \mathbb{R}^{100 \times 34}, y \in\{0,1\}$. Despite the dataset's small size, our network still obtains an average test accuracy of $92 \%$ across 350 random $\frac{2}{3}-\frac{1}{3}$ training-test splits. However, when we modify our test set to remove the 4 Iris components, average test accuracy falls to $81 \%$ with higher variance, suggesting the model learns to depend on Iris features and suffers without them. We verify that our explanations reveal this dependency and that regularizing them avoids it.

- Decoy MNIST: On the baseline MNST dataset [LeCun et al., 2010], our network obtains $98 \%$ train and 96\% test accuracy. However, in Decoy MNIST, images $x$ have $4 \times 4$ gray swatches in randomly chosen corners whose shades are functions of their digits $y$ in training (in particular, $255-25 y$ ) but are random in test. On this dataset, our model has a higher $99.6 \%$ train accuracy but a much lower 55\% test accuracy, indicating that the decoy rule misleads it. We verify that both gradient and LIME explanations let users detect this issue and that explanation regularization lets us overcome it.

Input gradients are consistent with sample-based methods such as LIME, and faster. On 20 Newsgroups (Figure 5), input gradients are less sparse but identify all of the same words in the document with similar weights. Note that input gradients also identify words outside the document that would affect the prediction if added.

On Decoy MNIST (Figure 6), both LIME and input gradients reveal that the model predicts 3 rather than 7 due to the color swatch in the corner. Because of their fine-grained resolution, input gradients sometimes better capture counterfactual behavior, where extending or adding lines outside of the digit to either reinforce it or transform it into another digit would 
Input gradients +soc.religion.christian +alt.atheism

From: USTS012@uabdpo.dpo.uab.edu

Subject: Should teenagers pick a church parents don't attend?

Organization: UTexas Mail-to-News Gateway

Lines: 13

Q. Should teenagers have the freedom to choose what church they go to?

My friends teenage kids do not like to go to church.

If left up to them they would sleep, but that's not an option.

They complain that they have no friends that go there, yet don't

attempt to make friends. They mention not respecting their Sunday

school teacher, and usually find a way to miss Sunday school but

do make it to the church service, (after their parents are thoroughly

disgusted) I might add. A never ending battle? It can just ruin your whole day if you let it.

Has anyone had this problem and how did it get resolved?

f.
LIME +soc.religion.christian +alt.atheism

From: USTS012@uabdpo.dpo.uab.edu

Subject: Should teenagers pick a church parents don't attend?

Organization: UTexas Mail-to-News Gateway

Lines: 13

Q. Should teenagers have the freedom to choose what church they go to?

My friends teenage kids do not like to go to church.

If left up to them they would sleep, but that's not an option.

They complain that they have no friends that go there, yet don't

attempt to make friends. They mention not respecting their Sunday

school teacher, and usually find a way to miss Sunday school but

do make it to the church service, (after their parents are thoroughly

disgusted) I might add. A never ending battle? It can just ruin your whole day if you let it.

Has anyone had this problem and how did it get resolved?

Figure 5: Words identified by LIME vs. gradients on an example from the atheism vs. Christianity subset of 20 Newsgroups. More examples are available at https://github.com/dtak/rrr. Words are blue if they support soc.religion.christian and orange if they support alt.atheism, with opacity equal to the ratio of the magnitude of the word's weight to the largest magnitude weight. LIME generates sparser explanations but the weights and signs of terms identified by both methods match closely. Note that both methods reveal some aspects of the model that are intuitive ("church" and "service" are associated with Christianity), some aspects that are not ("13" is associated with Christianity, "edu" with atheism), and some that are debatable ("freedom" is associated with atheism, "friends" with Christianity).

change the predicted probability (see also Figure 10). LIME, on the other hand, better captures the fact that the main portion of the digit is salient (because its super-pixel perturbations add and remove larger chunks of the digit).

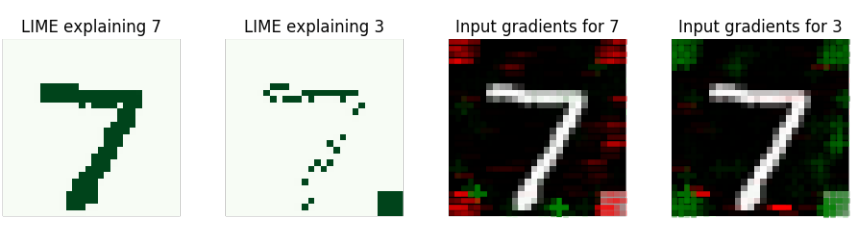

Figure 6: Input gradient explanations for Decoy MNIST vs. LIME, using the LIME image library [Ribeiro, 2016]. In this example, the model incorrectly predicts 3 rather than 7 because of the decoy swatch.
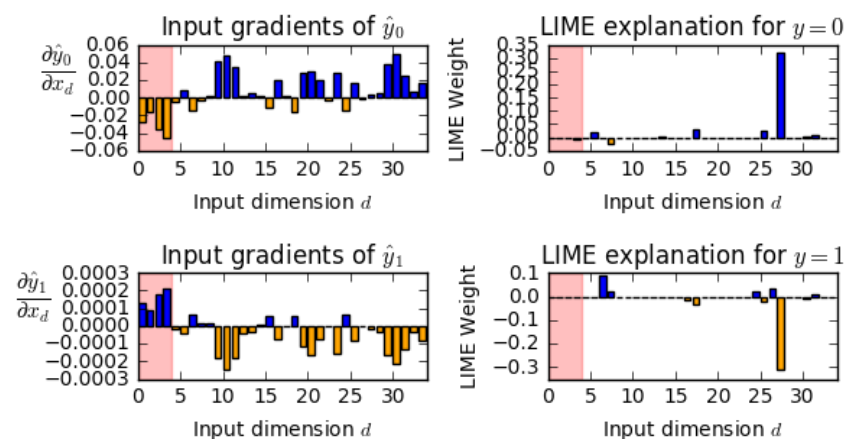

Figure 7: Iris-Cancer features identified by input gradients vs. LIME, with Iris features highlighted in red. Input gradient explanations are more faithful to the model. Note that most gradients change sign when switching between $\hat{y}_{0}$ and $\hat{y}_{1}$, and that the magnitudes of input gradients are different across examples, which provides information about examples' proximity to the decision boundary.
On Iris-Cancer (Figure 7), input gradients actually outperform LIME. We know from the accuracy difference that Iris features are important to the model's prediction, but LIME only identifies a single important feature, which is from the Breast Cancer dataset (even when we vary its perturbation strategy). This example, which is tabular and contains continuously valued rather categorical features, may represent a pathological case for LIME, which operates best when it can selectively mask a small number of meaningful chunks of its inputs to generate perturbed samples. For truly continuous inputs, it should not be surprising that explanations based on gradients perform best.

There are a few other advantages input gradients have over sample-based perturbation methods. On 20 Newsgroups, we noticed that for very long documents, explanations generated by the sample-based method LIME are often overly sparse (see Appendix C), and there are many words identified as significant by input gradients that LIME ignores. This may be because the number of features LIME selects must be passed in as a parameter beforehand, and it may also be because LIME only samples a fixed number of times. For sufficiently long documents, it is unlikely that sample-based approaches will mask every word even once, meaning that the output becomes increasingly nondeterministic — an undesirable quality for explanations. To resolve this issue, one could increase the number of samples, but that would increase the computational cost since the model must be evalutated at least once per sample to fit a local surrogate. Input gradients, on the other hand, only require on the order of one model evaluation total to generate an explanation of similar quality (generating gradients is similar in complexity to predicting probabilities), and furthermore, this complexity is based on the vector length, not the document length. This issue (underscored by Table 1) highlights some inherent scalability advantages input gradients enjoy over sample-based perturbation methods. 
Given annotations, input gradient regularization finds solutions consistent with domain knowledge. Another key advantage of using an explanation method more closely related to our model is that we can then incorporate explanations into our training process, which are most useful when the model faces ambiguities in how to classify inputs. We deliberately constructed the Decoy MNIST and Iris-Cancer datasets to have this kind of ambiguity, where a rule that works in training will not generalize to test. When we train our network on these confounded datasets, their test accuracy is better than random guessing, in part because the decoy rules are not simple and the primary rules not complex, but their performance is still significantly worse than on a baseline test set with no decoy

\begin{tabular}{|c|c|c|c|}
\hline & LIME & Gradients & Dimension of $x$ \\
\hline Iris-Cancer & $0.03 \mathrm{~s}$ & $0.000019 \mathrm{~s}$ & 34 \\
\hline Toy Colors & $1.03 \mathrm{~s}$ & $0.000013 \mathrm{~s}$ & 75 \\
\hline Decoy MNIST & $1.54 \mathrm{~s}$ & $0.000045 \mathrm{~s}$ & 784 \\
\hline 20 Newsgroups & $2.59 \mathrm{~s}$ & $0.000520 \mathrm{~s}$ & 5000 \\
\hline
\end{tabular}

Table 1: Gradient vs. LIME runtimes per explanation. Note that each method uses a different version of LIME; Iris-Cancer and Toy Colors use lime_tabular with continuous and quartile-discrete perturbation methods, respectively, Decoy MNIST uses lime_image, and 20 Newsgroups uses lime_text. Code was executed on a laptop and input gradient calculations were not optimized for performance, so runtimes are only meant to provide a sense of scale.

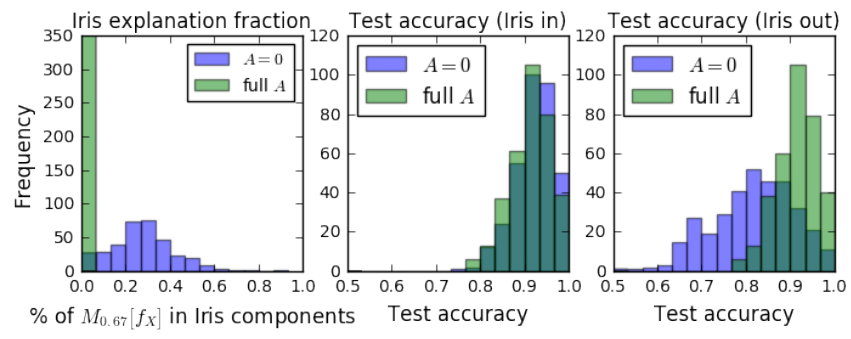

Figure 8: Overcoming confounds using explanation constraints on Iris-Cancer (over 350 random train-test splits). By default $(A=0)$, input gradients tend to be large in Iris dimensions, which results in lower accuracy when Iris is removed from the test set. Models trained with $A_{n d}=1$ in Iris dimensions (full $A$ ) have almost exactly the same test accuracy with and without Iris.

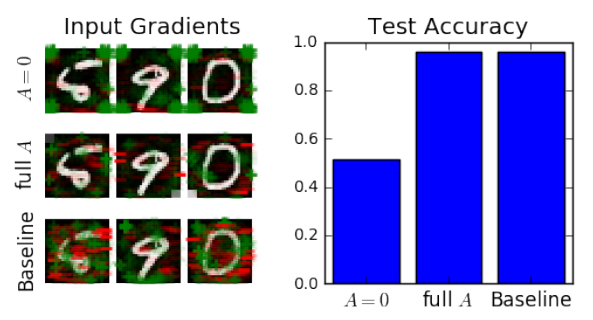

Figure 9: Training with explanation constraints on Decoy MNIST. Accuracy is low $(A=0)$ on the swatch color-randomized test set unless the model is trained with $A_{n d}=1$ in swatches (full $A$ ). In that case, test accuracy matches the same architecture's performance on the standard MNIST dataset (baseline). rules. By penalizing explanations we know to be incorrect using the loss function defined in Section 2.1, we are able to recover that baseline test accuracy, which we demonstrate in Figures 8 and 9.

When annotations are unavailable, our find-anotherexplanation method discovers diverse classifiers. As we
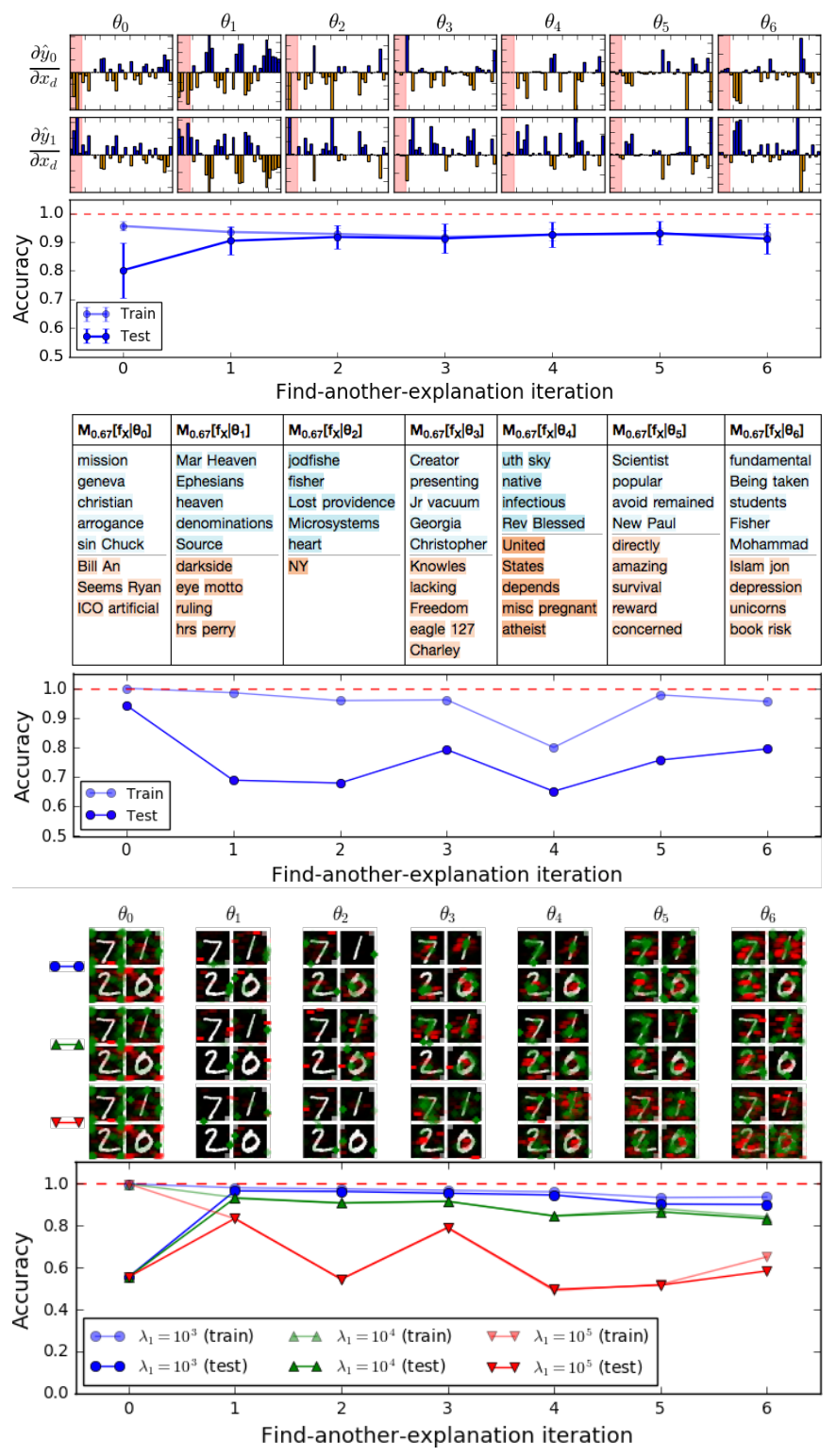

Figure 10: Find-another-explanation results on Iris-Cancer (top; errorbars show standard deviations across 50 trials), 20 Newsgroups (middle; blue supports Christianity and orange supports atheism, word opacity set to magnitude ratio), and Decoy MNIST (bottom, for three values of $\lambda_{1}$ with scatter opacity set to magnitude ratio cubed). Real-world datasets are often highly redundant and allow for diverse models with similar accuracies. On Iris-Cancer and Decoy MNIST, both explanations and accuracy results indicate we overcome confounds after 1-2 iterations without any prior knowledge about them encoded in $A$. 
saw with the Toy Color dataset, even if almost every row of $A$ is 0 , we can still benefit from explanation regularization (meaning practitioners can gradually incorporate these penalties into their existing models without much upfront investment). However, annotation is never free, and in some cases we either do not know the right explanation or cannot easily encode it. Additionally, we may be interested in exploring the structure of our model and dataset in a less supervised fashion. On real-world datasets, which are usually overdetermined, we can use find-another-explanation to discover $\theta$ s in shallower local minima that we would normally never explore. Given enough models right for different reasons, hopefully at least one is right for the right reasons.

Figure 10 shows find-another-explanation results for our three real-world datasets, with example explanations at each iteration above and model train and test accuracy below. For Iris-Cancer, we find that the initial iteration of the model heavily relies on the Iris features and has high train but low test accuracy, while subsequent iterations have lower train but higher test accuracy (with smaller gradients in Iris components). In other words, we spontaneously obtain a more generalizable model without a predefined $A$ alerting us that the first four features are misleading.

Find-another-explanation also overcomes confounds on Decoy MNIST, needing only one iteration to recover baseline accuracy. Bumping $\lambda_{1}$ too high (to the point where its term is a few orders of magnitude larger than the cross-entropy) results in more erratic behavior. Interestingly, in a process remniscent of distillation [Papernot et al., 2016], the gradients themselves become more evenly and intuitively distributed at later iterations. In many cases they indicate that the probabilities of certain digits increase when we brighten pixels along or extend their distinctive strokes, and that they decrease if we fill in unrelated dark areas, which seems desirable. However, by the last iteration, we start to revert to using decoy swatches in some cases.

On 20 Newsgroups, the words most associated with alt.atheism and soc.religion.christian change between iterations but remain mostly intuitive in their associations. Train accuracy mostly remains high while test accuracy is unstable.

For all of these examples, accuracy remains high even as decision boundaries shift significantly. This may because realworld data tends to contain significant redundancies.

\subsection{Limitations}

Input gradients provide faithful information about a model's rationale for a prediction but trade interpretability for efficiency. In particular, when input features are not individually meaningful to users (e.g. for individual pixels or word2vec components), input gradients may be difficult to interpret and $A$ may be difficult to specify. Additionally, because they can be 0 far from the decision boundary, they do not capture the idea of salience as well as other methods [Zeiler and Fergus, 2014; Sundararajan et al., 2016; Montavon et al., 2017; Bach et al., 2015; Shrikumar et al., 2016]. However, they are necessarily faithful to the model and easy to incorporate into its loss function. Input gradients are first-order linear approximations of the model; we might call them first-order explanations.

\section{Conclusions and Future Work}

We have demonstrated that training models with input gradient penalties makes it possible to learn generalizable decision logic even when our dataset contains inherent ambiguities. Input gradients are consistent with sample-based methods such as LIME but faster to compute and sometimes more faithful to the model, especially when our inputs are continous. Our find-another-explanation method can present a range of qualitatively different classifiers when such detailed annotations are not available, which may be useful in practice if we suspect each model is only right for the right reasons in certain regions. Our consistent results on several diverse datasets show that input gradients merit further investigation as scalable tools for optimizable explanations; there exist many options for further advancements such as weighted annotations $A$, different penalty norms (e.g. L1 regularization to encourage sparse gradients), and more general specifications of whether features should be positively or negatively predictive of specific classes for specific inputs.

Finally, our "right for the right reasons" approach may be of use in solving related problems, e.g. in maintaining robustness despite the presence of adversarial examples [Papernot et al., 2016], or seeing whether explanations and explanation constraints can further the goals of fairness, accountability, and transparency in machine learning (either by detecting indirect influence [Adler et al., 2016] or by constraining models to avoid it [Dwork et al., 2012; Zafar et al., 2016]). Building on our find-another-explanation results, another promising direction is to include humans in the loop to interactively guide models towards correct explanations. Overall, we feel that developing methods of ensuring that models are right for better reasons is essential to overcoming the inherent obstacles to generalization posed by ambiguities in real-world datasets.

\section{Acknowledgements}

FDV acknowledges support from DARPA W911NF-16-10561 and AFOSR FA9550-17-1-0155, and MCH thanks Oracle Labs for support. All authors thank Arjumand Masood, Sam Gershman, Paul Raccuglia, Mali Akmanalp, and the Harvard DTaK group for helpful discussions and insights.

\section{A Cross-Validation}

Most regularization parameters are selected to maximize accuracy on a validation set. However, when your training and validation sets share the same misleading confounds, validation accuracy may not be a good proxy for test accuracy. Instead, we recommend increasing the explanation regularization strength $\lambda_{1}$ until the cross-entropy and "right reasons" terms have roughly equal magnitudes (which corresponds to the region of highest test accuracy below). Intuitively, balancing the terms in this way should push our optimization away from cross-entropy minima that violate the explanation constraints specified in $A$ and towards ones that correspond 
to "better reasons." Increasing $\lambda_{1}$ too much makes the crossentropy term negligible. In that case, our model performs no better than random guessing.

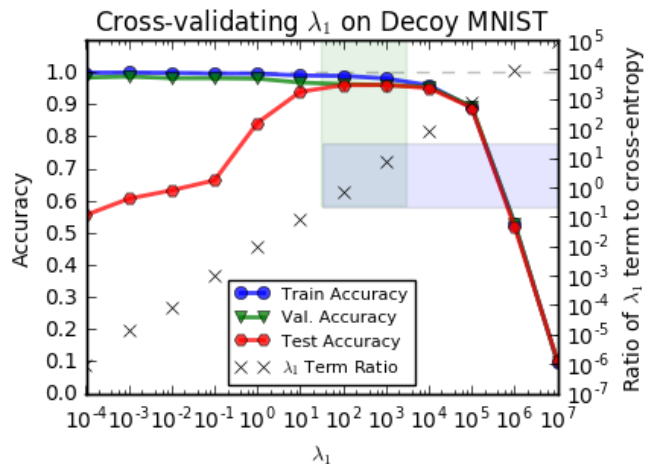

Figure 11: Cross-validating $\lambda_{1}$. The regime of highest accuracy (highlighted) is also where the initial cross-entropy and $\lambda_{1}$ loss terms have similar magnitudes. Exact equality is not required; being an order of magnitude off does not significantly affect accuracy.

\section{B Learning with Less Data}

It is natural to ask whether explanations can reduce data requirements. Here we explore that question on the Toy Color dataset using four variants of $A$ (with $\lambda_{1}$ chosen to match loss terms at each $N$ ).
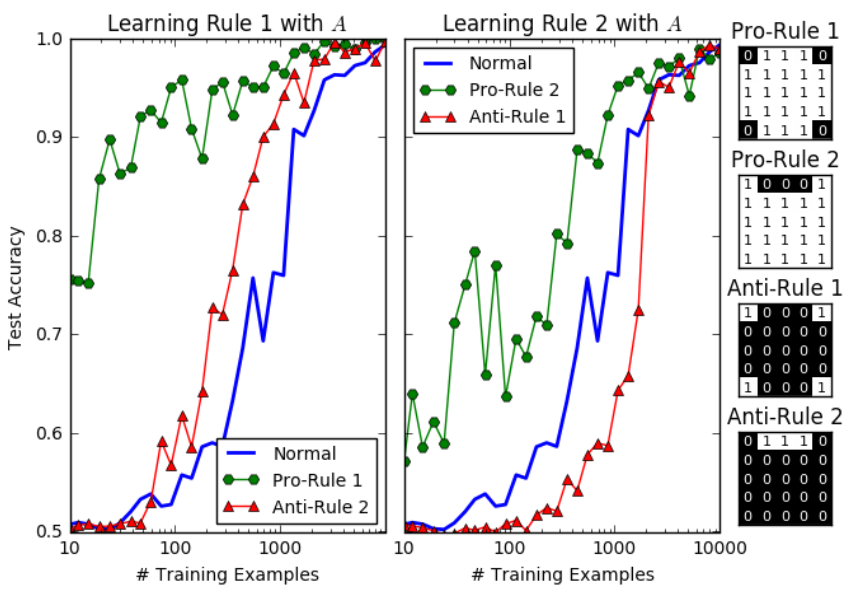

Figure 12: Explanation regularization can reduce data requirements.

We find that when $A$ is set to the Pro-Rule 1 mask, which penalizes all pixels except the corners, we reach $95 \%$ accuracy with fewer than 100 examples (as compared to $A=0$, where we need almost 10000). Penalizing the top-middle pixels (Anti-Rule 2) or all pixels except the top-middle (Pro-Rule 2) also consistently improves accuracy relative to data. Penalizing the corners (Anti-Rule 1), however, reduces accuracy until we reach a threshold $N$. This may be because the corner pixels can match in 4 ways, while the top-middle pixels can differ in $4 \cdot 3 \cdot 2=24$ ways, suggesting that Rule 2 could be inherently harder to learn from data and positional explanations alone.

\section{Longer 20 Newsgroups Examples}

\begin{tabular}{|c|c|}
\hline $\begin{array}{l}\text { Input gradients +alt.atheism } \\
\text { +soc.religion.christian }\end{array}$ & $\begin{array}{l}\text { LIME +alt.atheism } \\
\text { +soc.religion.christian }\end{array}$ \\
\hline From: darice@yoyo.cc.monash.edu.au (Fred Rice) & From: darice@yoyo.cc.monash.edu.au (Fred Rice) \\
\hline Subject: Re: Genocide is Caused by Atheism & Subject: Re: Genocide is Caused by Atheism \\
\hline Organization: Monash University, Melb., Australia. & Organization: Monash University, Melb., Australia. \\
\hline Lines: 19 & Lines: 19 \\
\hline $\begin{array}{l}\text { In <1993Apr19.140316.14872@cs.nott.ac.uk> } \\
\text { eczcaw@mips.nott.ac.uk (A.Wainwright) writes: }\end{array}$ & $\begin{array}{l}\text { In <1993Apr19.140316.14872@cs.nott.ac.uk> } \\
\text { eczcaw@mips.nott.ac.uk (A.Wainwright) writes: }\end{array}$ \\
\hline >In article & $>\ln$ article \\
\hline $\begin{array}{l}<1993 \text { Apr19.112706.26911@monu6.cc.monash.edu.au>, } \\
\text { darice@yoyo.cc.monash.edu.au (Fred Rice) writes: }\end{array}$ & $\begin{array}{l}\text {, <1993Apr19.112706.26911@monu6.cc.monash.edu.au>, } \\
\text { darice@yoyo.cc.monash.edu.au (Fred Rice) writes: }\end{array}$ \\
\hline $\begin{array}{l}>>\text { (Great respect or love for a particular person does } \\
\text { not equal a form of "theism".) }\end{array}$ & $\begin{array}{l}>>\text { (Great respect or love for a particular person does } \\
\text { not equal a form of "theism".) }\end{array}$ \\
\hline & \\
\hline$>>>$ Fred Rice & $>>>$ Fred Rice \\
\hline$>\mid>$ darice@yoyo.cc.monash.edu.au & $>\mid>$ darice@yoyo.cc.monash.edu.au \\
\hline$>$ Hmm. What about Jesus? & $>$ Hmm. What about Jesus? \\
\hline $\begin{array}{l}\text { Sure, a person could have great respect for Jesus and } \\
\text { yet be an atheist. (Having great respect for Jesus does } \\
\text { not necessarily mean that one has to follow the } \\
\text { Christian [or Muslim] interpretation of his life.) }\end{array}$ & $\begin{array}{l}\text { Sure, a person could have great respect for Jesus and } \\
\text { yet be an atheist. (Having great respect for Jesus does } \\
\text { not necessarily mean that one has to follow the } \\
\text { Christian [or Muslim] interpretation of his life.) }\end{array}$ \\
\hline $\begin{array}{l}\text { Fred Rice } \\
\text { darice@yoyo.cc.monash.edu.au }\end{array}$ & $\begin{array}{l}\text { Fred Rice } \\
\text { darice@yoyo.cc.monash.edu.au }\end{array}$ \\
\hline $\begin{array}{l}\text { Input gradients +soc.religion.christian } \\
\text { talt.atheism }\end{array}$ & $\begin{array}{l}\text { LIME +soc.religion.christian } \\
\text { +alt.atheism }\end{array}$ \\
\hline $\begin{array}{l}\text { From: creps@lateran.ucs.indiana.edu (Stephen A. } \\
\text { Creps) }\end{array}$ & $\begin{array}{l}\text { From: creps@|ateran.ucs.indiana.edu (Stephen A. } \\
\text { Creps) }\end{array}$ \\
\hline Subject: Re: What WAS the immaculate conception & Subject: Re: What WAS the immaculate conception \\
\hline Organization: Indiana University & Organization: Indiana University \\
\hline Lines: 28 & Lines: 28 \\
\hline In article & In article \\
\hline $\begin{array}{l}\text { <May.14.02.11.19.1993.25177@athos.rutgers.edu> } \\
\text { seanna@bnr.ca (Seanna (S.M.) Watson) wrote: }\end{array}$ & $\begin{array}{l}\text { <May.14.02.11.19.1993.25177@athos.rutgers.edu> } \\
\text { seanna@bnr.ca (Seanna (S.M.) Watson) wrote: }\end{array}$ \\
\hline $\begin{array}{l}\text { >I have quite a problem with the idea that Mary never } \\
\text { committed a sin. }\end{array}$ & $\begin{array}{l}>1 \text { have quite a problem with the idea that Mary never } \\
\text { committed a sin. }\end{array}$ \\
\hline $\begin{array}{l}\text { >Was Mary fully human? If it is possible for God to } \\
\text { miraculously make }\end{array}$ & $\begin{array}{l}>\text { Was Mary fully human? If it is possible for God to } \\
\text { miraculously make }\end{array}$ \\
\hline $\begin{array}{l}>\text { a person free of original sin, and free of committing } \\
\sin \text { their whole }\end{array}$ & $\begin{array}{l}\text { >a person free of original sin, and free of committing } \\
\text { sin their whole }\end{array}$ \\
\hline $\begin{array}{l}\text { >life, then what is the purpose of the Incarnation of } \\
\text { Jesus? Why can't }\end{array}$ & $\begin{array}{l}\text { life, then what is the purpose of the Incarnation of } \\
\text { Jesus? Why can't }\end{array}$ \\
\hline $\begin{array}{l}>\text { God just repeat the miracle done for Mary to make all } \\
\text { the rest of us } \\
>\text { sinless, without the need for repentance and } \\
\text { salvation and all that? }\end{array}$ & $\begin{array}{l}>\text { God just repeat the miracle done for Mary to make all } \\
\text { the rest of us } \\
>\text { sinless, without the need for repentance and } \\
\text { salvation and all that? }\end{array}$ \\
\hline $\begin{array}{l}\text { Yes, Mary is fully human. However, that does not imply } \\
\text { that she was just as subject to sin as we are. Catholic } \\
\text { doctrine says that man's nature is good (Gen } 1: 31 \text { ), } \\
\text { but is damaged by Original Sin (Rom } 5: 12-16) \text {. In that } \\
\text { case, being undamaged by Original Sin, Mary is more } \\
\text { fully human than any of the rest of us. }\end{array}$ & $\begin{array}{l}\text { Yes, Mary is fully human. However, that does not imply } \\
\text { that she was just as subject to sin as we are. Catholic } \\
\text { doctrine says that man's nature is good (Gen } 1: 31 \text {, } \\
\text { but is damaged by Original Sin (Rom } 5: 12-16) \text {. In that } \\
\text { case, being undamaged by Original } \operatorname{Sin}, \text { Mary is more } \\
\text { fully human than any of the rest of us. }\end{array}$ \\
\hline You ask why God cannot "repeat the miracle" of & You ask why God cannot "repeat the miracle" of \\
\hline $\begin{array}{l}\text { Mary's preservation from Original Sin. A better way to } \\
\text { phrase it would be "why _did_ He not" do it that way, } \\
\text { but you misunderstand how Mary's salvation was } \\
\text { obtained. Like ours, the Blessed Virgin Mary's } \\
\text { salvation was obtained through the merits of the }\end{array}$ & $\begin{array}{l}\text { Mary's preservation from Original Sin. A better way to } \\
\text { phrase it would be "why_did_ He not" do it that way, } \\
\text { but you misunderstand how Mary's salvation was } \\
\text { obtained. Like ours, the Blessed Virgin Mary's } \\
\text { salvation was obtained through the merits of the }\end{array}$ \\
\hline $\begin{array}{l}\text { Sacrifice of Christ on the Cross. However, as God is } \\
\text { not bound by time, which is His creation, God is free } \\
\text { to apply His Sacrifice to anyone at any time, even if } \\
\text { that person lived before Christ came to Earth, from our } \\
\text { time-bound perspective. Therefore, Christ's Death and }\end{array}$ & $\begin{array}{l}\text { Sacrifice of Christ on the Cross. However, as God is } \\
\text { not bound by time, which is His creation, God is free } \\
\text { to apply His Sacrifice to anyone at any time, even if } \\
\text { that person lived before Christ came to Earth, from our } \\
\text { time-bound perspective. Therefore, Christ's Death and }\end{array}$ \\
\hline $\begin{array}{l}\text { Resurrection still served a necessary purpose, and } \\
\text { were necessary even for Mary's salvation. }\end{array}$ & $\begin{array}{l}\text { Resurrection still served a necessary purpose, and } \\
\text { were necessary even for Mary's salvation. }\end{array}$ \\
\hline & \\
\hline $\begin{array}{l}\text { Steve Creps, Indiana University } \\
\text { creps@lateran.ucs.indiana.edu }\end{array}$ & $\begin{array}{l}\text { Steve Creps, Indiana University } \\
\text { creps@lateran.ucs.indiana.edu }\end{array}$ \\
\hline
\end{tabular}

Figure 13: Longer 20 Newsgroups examples. Blue supports the predicted label, orange opposes it, and opacity ${ }_{i}=\left|w_{i}\right| / \max |w|$. LIME and input gradients never disagree, but gradients may provide a fuller picture of the model's behavior because of LIME's limits on features and samples (especially for long documents). 


\section{References}

[Adler et al., 2016] Philip Adler, Casey Falk, Sorelle A Friedler, Gabriel Rybeck, Carlos Scheidegger, Brandon Smith, and Suresh Venkatasubramanian. Auditing black-box models by obscuring features. arXiv preprint arXiv:1602.07043, 2016.

[Bach et al., 2015] Sebastian Bach, Alexander Binder, Grégoire Montavon, Frederick Klauschen, Klaus-Robert Müller, and Wojciech Samek. On pixel-wise explanations for non-linear classifier decisions by layer-wise relevance propagation. PloS one, 10(7):e0130140, 2015.

[Baehrens et al., 2010] David Baehrens, Timon Schroeter, Stefan Harmeling, Motoaki Kawanabe, Katja Hansen, and Klaus-Robert Müller. How to explain individual classification decisions. Journal of Machine Learning Research, 11(Jun):1803-1831, 2010.

[Caruana et al., 2015] Rich Caruana, Yin Lou, Johannes Gehrke, Paul Koch, Marc Sturm, and Noemie Elhadad. Intelligible models for healthcare: Predicting pneumonia risk and hospital 30-day readmission. In Proceedings of the 21th ACM SIGKDD International Conference on Knowledge Discovery and Data Mining, pages 1721-1730. ACM, 2015.

[Donahue and Grauman, 2011] Jeff Donahue and Kristen Grauman. Annotator rationales for visual recognition. In 2011 International Conference on Computer Vision, pages 1395-1402. IEEE, 2011.

[Doshi-Velez and Kim, 2017] Finale Doshi-Velez and Been Kim. Towards a rigorous science of interpretable machine learning. arXiv preprint arXiv:1702.08608, 2017.

[Drucker and Le Cun, 1992] Harris Drucker and Yann Le Cun. Improving generalization performance using double backpropagation. IEEE Transactions on Neural Networks, 3(6):991-997, 1992.

[Dwork et al., 2012] Cynthia Dwork, Moritz Hardt, Toniann Pitassi, Omer Reingold, and Richard Zemel. Fairness through awareness. In Proceedings of the 3rd Innovations in Theoretical Computer Science Conference, pages 214-226. ACM, 2012.

[Fong and Vedaldi, 2017] Ruth Fong and Andrea Vedaldi. Interpretable explanations of black boxes by meaningful perturbation. arXiv preprint arXiv:1704.03296, 2017.

[Hechtlinger, 2016] Yotam Hechtlinger. Interpretation of prediction models using the input gradient. arXiv preprint arXiv:1611.07634, 2016.

[Keil, 2006] Frank C Keil. Explanation and understanding. Annu. Rev. Psychol., 57:227-254, 2006.

[Kingma and Ba, 2014] Diederik Kingma and Jimmy Ba. Adam: A method for stochastic optimization. arXiv preprint arXiv:1412.6980, 2014.

[LeCun et al., 2010] Yann LeCun, Corinna Cortes, and Christopher J.C. Burges. The MNIST database of handwritten digits. http://yann. lecun.com/exdb/mnist/, 2010.

[Lei et al., 2016] Tao Lei, Regina Barzilay, and Tommi Jaakkola. Rationalizing neural predictions. arXiv preprint arXiv:1606.04155, 2016.

[Li et al., 2015] Jiwei Li, Xinlei Chen, Eduard Hovy, and Dan Jurafsky. Visualizing and understanding neural models in NLP. arXiv preprint arXiv:1506.01066, 2015.

[Li et al., 2016] Jiwei Li, Will Monroe, and Dan Jurafsky. Understanding neural networks through representation erasure. arXiv preprint arXiv:1612.08220, 2016.

[Lichman, 2013] M. Lichman. UCI machine learning repository. http://archive.ics.uci.edu/ml, 2013.
[Lipton, 2016] Zachary C Lipton. The mythos of model interpretability. arXiv preprint arXiv:1606.03490, 2016.

[Lundberg and Lee, 2016] Scott Lundberg and Su-In Lee. An unexpected unity among methods for interpreting model predictions. arXiv preprint arXiv:1611.07478, 2016.

[Mclaurin et al., 2017] Dougal Mclaurin, David Duvenaud, and Matt Johnson. Autograd. https://github.com/HIPS/ autograd, 2017.

[Montavon et al., 2017] Grégoire Montavon, Sebastian Lapuschkin, Alexander Binder, Wojciech Samek, and Klaus-Robert Müller. Explaining nonlinear classification decisions with deep Taylor decomposition. Pattern Recognition, 65:211-222, 2017.

[Papernot et al., 2016] Nicolas Papernot, Patrick McDaniel, Xi Wu, Somesh Jha, and Ananthram Swami. Distillation as a defense to adversarial perturbations against deep neural networks. In Security and Privacy (SP), 2016 IEEE Symposium on, pages 582597. IEEE, 2016.

[Ribeiro et al., 2016] Marco Tulio Ribeiro, Sameer Singh, and Carlos Guestrin. Why should I trust you?: Explaining the predictions of any classifier. In Proceedings of the 22nd ACM SIGKDD International Conference on Knowledge Discovery and Data Mining, pages 1135-1144. ACM, 2016.

[Ribeiro, 2016] Marco Tulio Ribeiro. LIME. https ://github. com/marcotcr/lime, 2016.

[Selvaraju et al., 2016] Ramprasaath R Selvaraju, Abhishek Das, Ramakrishna Vedantam, Michael Cogswell, Devi Parikh, and Dhruv Batra. Grad-CAM: Why did you say that? arXiv preprint arXiv:1611.07450, 2016.

[Shrikumar et al., 2016] Avanti Shrikumar, Peyton Greenside, Anna Shcherbina, and Anshul Kundaje. Not just a black box: Learning important features through propagating activation differences. arXiv preprint arXiv:1605.01713, 2016.

[Shrikumar et al., 2017] Avanti Shrikumar, Peyton Greenside, and Anshul Kundaje. Learning important features through propagating activation differences. arXiv preprint arXiv:1704.02685, 2017.

[Simonyan et al., 2013] Karen Simonyan, Andrea Vedaldi, and Andrew Zisserman. Deep inside convolutional networks: Visualising image classification models and saliency maps. arXiv preprint arXiv:1312.6034, 2013.

[Singh et al., 2016] Sameer Singh, Marco Tulio Ribeiro, and Carlos Guestrin. Programs as black-box explanations. arXiv preprint arXiv:1611.07579, 2016.

[Sundararajan et al., 2016] Mukund Sundararajan, Ankur Taly, and Qiqi Yan. Gradients of counterfactuals. arXiv preprint arXiv:1611.02639, 2016.

[Zafar et al., 2016] Muhammad Bilal Zafar, Isabel Valera, Manuel Gomez Rodriguez, and Krishna P Gummadi. Fairness beyond disparate treatment \& disparate impact: Learning classification without disparate mistreatment. arXiv preprint arXiv:1610.08452, 2016.

[Zaidan et al., 2007] Omar Zaidan, Jason Eisner, and Christine D Piatko. Using "annotator rationales" to improve machine learning for text categorization. In HLT-NAACL, pages 260-267, 2007.

[Zeiler and Fergus, 2014] Matthew D Zeiler and Rob Fergus. Visualizing and understanding convolutional networks. In European conference on computer vision, pages 818-833. Springer, 2014.

[Zhang et al., 2016] Ye Zhang, Iain Marshall, and Byron C Wallace. Rationale-augmented convolutional neural networks for text classification. arXiv preprint arXiv:1605.04469, 2016. 